\title{
EDITORIAL
}

\section{La epidemia del tabaquismo en las Américas}

E 1 consumo de tabaco es un problema con importantes repercusiones sanitarias, ambientales, sociales, políticas y económicas para la región. ${ }^{1}$ La solución a este problema se logrará únicamente con la participación comprometida de nuestros gobiernos y con la participación de la comunidad internacional para poner en marcha intervenciones multisectoriales que armonicen las actividades de control, tanto en el ámbito nacional como en el continental. ${ }^{2}$ En este documento se presenta el problema del tabaquismo en el continente americano, y se mencionan algunos pasos que deberán seguir los gobiernos locales y la comunidad internacional para combatir la epidemia global.

\section{Contexto en las A méricas}

El consumo de tabaco es uno de los retos de salud pública más importantes para la región de las Américas, ya que causa al menos 845000 defunciones por año. ${ }^{1}$ El consumo de tabaco produce enfermedades mortales y discapacitantes. Se considera que es causa, o causa probable, de más de 25 enfermedades entre las que destacan el cáncer de pulmón, la enfermedad isquémica cardiovascular y el enfisema pulmonar. Se estima que la mitad de todos los fumadores crónicos morirán prematuramente y que en promedio perderán 20 a 25 años de su vida, e impondrán un importante costo social y económico a sus familias y a los sistemas médicos gubernamentales. En el ámbito mundial se calcula que el consumo de tabaco es responsable de la muerte prematura de más de $3.5 \mathrm{mi}$ llones de personas por año., ${ }^{2,3}$

Si bien los indicadores de la epidemia de tabaquismo muestran variaciones de acuerdo con cada subregión americana, todos ellos coinciden en identificar la epidemia de tabaquismo como un gran problema de salud pública en el continente, que requiere atención inmediata y concertada entre los gobiernos. En- el continente americano cerca de la tercera parte de la población informa consumo cotidiano de tabaco. ${ }^{4}$ En los países del Cono Sur se observan los consumos más elevados y cierta tendencia en los indicadores que sugieren estabilidad en su consumo. Los países andinos ocupan el segundo lugar de consumo de tabaco y tienden, asimismo, hacia la estabilización de ese consumo. La subregión mexicana presenta un índice de consumo similar a los países de la subregión andina, y en los países de América Central y el Caribe se reportan los índices más bajos de consumo de tabaco. En contraste con otras subregiones, en Estados Unidos de América y Canadá se observan cambios en los indicadores que muestran una reducción considerable en el consumo de tabaco a nivel de población general, y una tendencia que se mantiene a la baja.

En relación con la distribución del consumo por género, se observa que en la Región de las Américas, es más frecuente en el sexo masculino; sin embargo, datos recientes indican que el consumo entre las mujeres tiende a aumentar y a ser similar en magnitud al que se observa entre los hombres. Esto es particularmente alarmante ya que el consumo de tabaco en las mujeres gestantes pone en riesgo el bienestar del producto, aumenta considerablemente el riesgo de bajo peso al nacer y muerte infantil, y disminuye considerablemente las posibilidades de un desarrollo infantil pleno. ${ }^{4}$

Al igual que las mujeres, ${ }^{5}$ los jóvenes ${ }^{6}$ también han sido blanco comercial de las campañas de promoción del tabaquismo realizadas por las compañías tabacaleras. Estudios recientes indican una tendencia importante hacia que los jóvenes inicien a edades más tempranas el consumo de cigarrillos. ${ }^{4,6}$ En el mundo se estima que diariamente se inician como fumadores cerca de 100000 jóvenes. En la Región, una proporción considerablemente alta de los consumidores de tabaco reporta haberse iniciado entre los 12 y los 18 
años de edad. ${ }^{4}$ Se estima que aproximadamente $75 \%$ de los fumadores iniciaron antes de alcanzar los 19 años. El consumo de tabaco por la población joven tiende a reflejar la variación y consumo observada entre los adultos, y es en los países del Cono Sur en donde se encuentran las prevalencias más elevadas, y en la subregión de América Central y el Caribe, las menores.

La exposición al humo ambiental del tabaco es también un problema importante, aunque poco caracterizado en la región. ${ }^{78}$ Se ha demostrado que el humo de tabaco contiene una gran cantidad de productos químicos, entre los que destacan al menos 40 carcinógenos reconocidos y cantidades importantes de monóxido de carbono. Según la Agencia de Protección del Medio Ambiente, se estima que en California ocurren entre 3000 y 8000 muertes por año en no fumadores, debido a la exposición involuntaria al humo de tabaco. ${ }^{8}$ A este respecto, es importante reconocer que la población infantil es particularmente vulnerable a la exposición al humo ambiental del tabaco, y por ello se requieren acciones precisas para protegerla. Los niños tienen un metabolismo y tasa de respiración que son de mayor intensidad, razón por la cual la dosis que reciben de humo de tabaco es considerablemente mayor; esto propicia un aumento en la frecuencia de infecciones respiratorias y del oído. Finalmente, los niños tienen menos opciones que los adultos para evitar voluntariamente la exposición, ya que les es más difícil salir de o evitar espacios contaminados con humo de tabaco.

Un aspecto importante que limita el control de la epidemia es la falta de información que prevalece en relación con los daños que ocasiona el consumo de tabaco en la economía de los países. ${ }^{9}$ Estimaciones realizadas en los países de ingreso alto indican que la atención de salud relacionada con el tabaco representa entre 6 y $15 \%$ del total de los gastos anuales en salud. ${ }^{10,11}$ En los Estados Unidos de América el costo de las enfermedades asociadas con el tabaco se ha estimado entre $\$ 50$ y $\$ 70$ mil millones de dólares, lo que equivale a 6 o $12 \%$ de los costos totales para ese país. ${ }^{10}$ Es importante considerar que muy probablemente estas estimaciones no son extrapolables a las de los países de ingreso medio y bajo de la Región, cuyas epidemias de enfermedades relacionadas con el tabaco se encuentran en etapas más tempranas, pero sí indican los grandes costos que se podrían enfrentar en ella de no intervenir decididamente, instrumentando mecanismos de control para disminuir el uso de tabaco en la población general y para desalentar a los jóvenes a iniciar el consumo de tabaco. Sin embargo, los pocos da- tos reportados a la fecha indican un costo considerable. Por ejemplo, para Venezuela se ha estimado que las enfermedades atribuibles al tabaco imponen una carga económica equivalente a casi $0.5 \%$ del producto interno bruto. ${ }^{12}$

\section{Recomendaciones}

Para lograr el control efectivo del tabaquismo en la Región será imperativo promover un ambiente favorable tanto en lo político como en lo social. ${ }^{12}$ Para alcanzar esto, es necesario abordar el problema con una visión en la que se reconozca a la nicotina en el tabaco como una droga, y al cigarrillo como un instrumento de dosificación, que pueden ser controlados con políticas públicas en los diferentes sectores. Es importante también incorporar la visión de que los ámbitos de acción nacional e internacional son igualmente importantes. Para arribar a un control efectivo del tabaquismo será indispensable desarrollar políticas de control multisectoriales al interior de los países, así como los acuerdos internacionales necesarios para reforzar y garantizar el éxito de las políticas implantadas al interior de aquéllos.

Con el fin de lograr un control efectivo de la adicción en la región es necesario trabajar con la población en general, aumentando el nivel de información sobre los daños que ocasiona el tabaco, ya que a medida que las personas estén mejor informadas podrán realizar las decisiones más apropiadas para conservar su salud. Por otro lado, se deberá trabajar aumentando la capacidad de los gobiernos para desarrollar intervenciones económicas, legales y de salud pública, que sienten las bases que empoderen a los gobiernos para mejor proteger a la población de esta pandemia. ${ }^{12}$

A continuación se sugieren algunas líneas estratégicas.

1. Implantar una política fiscal saludable con las siguientes líneas de acción

- Aumentar las tasas impositivas a los cigarros con y sin filtro, nacionales e importados, logrando una homogeneidad de precios en la Región

- Aumentar en forma gradual, pero continua, las tasas impositivas a toda la producción y venta de tabaco a niveles que desalienten su consumo

- Dedicar una parte proporcional de la recaudación fiscal asociada con el tabaco para apoyar la prevención y control de la adicción. 
2. Instrumentar acciones nacionales e internacionales, para vigilar y prevenir el contrabando y venta ilegal de cigarrillos u otros productos nocivos del tabaco

3. Promulgar leyes y reglamentos para proteger a la población no fumadora, promoviendo los espacios libres de humo de tabaco

4. Aumentar la difusión de los efectos que tiene el humo de tabaco en la población fumadora y no fumadora

5. Implantar acciones para disminuir el acceso al tabaco de la población menor de edad y de los jóvenes

- Se vigilará la aplicación de reglamentos estatales para la no venta de cigarrillos, a menores de edad

- Se aumentarán los precios de los cigarrillos y productos del tabaco, mediante un incremento en el impuesto

- Se instrumentarán programas educativos y de información para que los jóvenes conozcan mejor los efectos del tabaquismo.

6. Implantar acciones para fortalecer y hacer accesibles el diagnóstico y el tratamiento para revertir la adicción a la nicotina

7. Eliminar completamente la publicidad en los medios de comunicación masiva

8. Auxiliar a los agricultores para introducir otros cultivos que desplacen al tabaco

9. Aumentar la capacidad institucional (gubernamental y no gubernamental) para el control del tabaquismo mediante

- Adecuación y vigilancia del cumplimiento de las normas

- Vigilancia epidemiológica sobre el curso de la epidemia

- Investigación aplicada para disminuir el consumo de tabaco

- Evaluación de los programas nacionales y subregionales antitabaco.

10. Difundir el conocimiento relacionado con el curso de la epidemia y el impacto de las acciones realizadas
- Identificando indicadores de operación, de efecto y de impacto para evaluaciones en el corto y mediano plazo

- Apoyando las acciones de investigación encaminadas al diseño y evaluación de programas educativos y de prevención.

11. Conseguir la decisión política al más alto nivel de los Estados miembros por medio de la cual se consiga

- La plena participación y compromiso de las instituciones en el desarrollo del Convenio Marco para el Control del Tabaco

- Movilizar los recursos financieros indispensables para apoyar las acciones en el ámbito nacional e internacional acordes con la magnitud que este problema reclama para la salud pública de la Región.

Para fortalecer las actividades de control del tabaquismo en la Región la Oficina Panamericana de la Salud debe intensificar las actividades de cooperación técnica, así como el establecimiento del Centro Panamericano para el desarrollo de recursos humanos, coordinación de la investigación y cooperación técnica con los gobiernos miembros, para lograr un sistema de manejo integrado del consumo de tabaco y la adicción a la nicotina.

Dr. Jaime Sepúlveda*

\section{Referencias}

1.Tabaco 0 salud: situación en las Américas. Un informe de la 0 rganización Panamericana de la Salud. Publicación Científica N úm. 536, 1992.

2. Jha P, Chaloupka F. Curbing the epidemic: Governments and the economics of to bacco control. W ashington, D.C.: The W orld Bank, 1999.

3. Peto R, López AD, Boreham J,Thun M, H eath C. Mortality from smoking in developed countries, 1950-2000. 0 xford, England: 0 xford University Press, 1994.

4. 0 rganización Panamericana de la Salud. 0 ficina Regional de la 0 rganización Mundial de la Salud. El tabaquismo en América Latina, Estados Unidos y Canadá (Periodo 1990-1999). 2000.

5. Samet JM, Yoon Soon-Young, ed.W omen and the to bacco epidemic challenges for the 21st century. G inebra:TheW orld H ealth 0 rganization, 2001.

* Director General del Instituto Nacional de Salud Pública. 
6.W arren W, Riley L,A sma S, Eriksen M, G reen L, Blanton C et al. Tobacco use by youth:A surveillance report from the global youth to bacco survey. Ginebra:W orld Health 0 rganization, 2000.

7. California Environmental Protection A gency. 0 ffice of Environmental $\mathrm{H}$ ealth $\mathrm{H}$ azards Assessment. Health effects of exposure to environmental to bacco smoke. Final Report, 1997.

8. California Environmental Protection A gency. Health Effects of Exposure to Environmental Tobacco Smoke: The Report of the California Environmental Protection A gency. Bethesda (MD ): N ational Institutes of Health, 1999.

9. Meneses-G onzález F, Márquez-Serrano M, Sepúlveda-A mor J, Hernández-Avila M. Gasto en tabaco. En: Peña-C orona Gutiérrez MC, Kuri-Morales P,Tapia-C onyer R, comp. El consumo de Tabaco en México y Encuesta
N acional de Adicciones 1998 (Tabaco). México, D.F.: Secretaría de Salud, 2000:33-50

10. Centers for Disease Control and Prevention. Medical-care expenditures attributable to cigarette smoking in the U nited States, 1993. MMW R Morb Mortal W kly Rep 1994;43:469-472.

11. U.S. Department of Health and Human Services. Reducing Tobacco Use:A Report of the Surgeon G eneral. Atlanta (GA): U.S. D epartment of Health and Human Services, Centers for D isease Control and Prevention, $\mathrm{N}$ ational $\mathrm{C}$ enter for $\mathrm{Chronic} \mathrm{D}$ isease Prevention and Health Promotion, 0 ffice on Smoking and Health, 2000.

12. Chaloupka FJ, Hu T-W, W arner KE, van der Merwe R, Yurekli A. The taxation of tobacco products. En:Jha P, C halo upka FJ, ed.Tobacco C ontrol in Developing Countries. 0 xford: 0 xford U niversity Press. In press. 